(1)

CrossMark

\title{
Biomarkers of carcinogenesis and tumour growth in patients with cutaneous melanoma and obstructive sleep apnoea
}

\author{
Fernando Santamaria-Martos (1) 1 , Ivan Benítez ${ }^{1}$, Cristina Girón ${ }^{1}$, \\ Ferran Barbé ${ }^{1,2}$, Miguel-Angel Martínez-García ${ }^{3}$, Luis Hernández ${ }^{4}$, \\ Josep M. Montserrat ${ }^{5}$, Eduardo Nagore ${ }^{6}$, Antonio Martorell ${ }^{7}$, \\ Francisco Campos-Rodriguez ${ }^{8}$, Jaime Corral ${ }^{9}$, Valentin Cabriada (10 ${ }^{10}$, \\ Jorge Abad $^{11}$, Olga Mediano ${ }^{12}$, Maria F. Troncoso ${ }^{13}$, Irene Cano-Pumarega (1) ${ }^{14}$, \\ Ana Maria Fortuna Gutierrez ${ }^{15}$, Trinidad Diaz-Cambriles ${ }^{2,16}$, \\ Maria Somoza-Gonzalez ${ }^{17}$, Isaac Almendros ${ }^{2,18}$, Ramon Farre ${ }^{2,18}$, David Gozal $^{19}$ \\ and Manuel Sánchez-de-la-Torre (10 ${ }^{1,2}$, on behalf of The Spanish Sleep Network
}

@ERSpublications

In patients with cutaneous melanoma, OSA is associated with increased expression of a biomarker of tumorigenesis http://ow.ly/mLo430i9Z8j

Cite this article as: Santamaria-Martos F, Benítez I, Girón C, et al. Biomarkers of carcinogenesis and tumour growth in patients with cutaneous melanoma and obstructive sleep apnoea. Eur Respir J 2018; 51: 1701885 [https://doi.org/10.1183/13993003.01885-2017].

ABSTRACT The goal of this study was to assess the relationship between the severity of obstructive sleep apnoea (OSA) and the levels of carcinogenesis- and tumour growth-related biomarkers in patients with cutaneous melanoma.

This multicentre observational study included patients who were newly diagnosed with melanoma. The patients were classified as non-OSA (apnoea-hypopnoea index (AHI) 0-5 events.h ${ }^{-1}$ ), mild OSA (AHI 515 events $\cdot \mathrm{h}^{-1}$ ) and moderate-severe OSA (AHI $>15$ events $\cdot \mathrm{h}^{-1}$ ). ELISAs were performed to analyse the serum levels of hypoxia- and tumour adhesion-related biomarkers (vascular endothelial growth factor (VEGF), interleukin (IL)-8, intracellular adhesion molecule (ICAM) and vascular cell adhesion molecule (VCAM)-1) and markers of tumour aggressiveness (S100 calcium-binding protein B (S100B) and melanoma inhibitory activity (MIA)). A logistic model adjusted for age, sex and body mass index was fitted to each biomarker, and the AHI served as the dependent variable.

360 patients were included (52.2\% male, median (interquartile range) age 55.5 (43.8-68.0) years and AHI $8.55(2.8-19.5)$ events $\left.\cdot h^{-1}\right)$. The levels of VEGF, IL-8, ICAM-1, S100B and MIA were not related to the severity of OSA. The levels of VCAM-1 were higher in patients with OSA than those without OSA (mild OSA: odds ratio (OR) 2.07, $\mathrm{p}=0.021$; moderate-severe OSA: OR 2.35, $\mathrm{p}=0.013$ ).

In patients with cutaneous melanoma, OSA was associated with elevated circulating levels of VCAM-1 that could indicate the contribution of OSA in tumorigenesis via integrin-based adhesion.

This article has supplementary material available from erj.ersjournals.com

Received: Sept 152017 | Accepted after revision: Jan 292018

Support statement: The project is supported by FIS 2016/01772 from the Spanish Ministry of Health, Sociedad Española de Neumología y Cirugía Torácica (SEPAR) and Sociedad Valenciana de Neumología. F. Santamaria-Martos is the recipient of a predoctoral fellowship from "AGAUR-University of Lleida". Funding information for this article has been deposited with the Crossref Funder Registry.

Conflict of interest: None declared.

Copyright OERS 2018 
Affiliations: ${ }^{1}$ Group of Translational Research in Respiratory Medicine, Hospital Universitari Arnau de Vilanova y Santa Maria, IRB Lleida, Lleida, Spain. ${ }^{2}$ Centro de Investigación Biomédica en Red de Enfermedades Respiratorias (CIBERES), Madrid, Spain. ${ }^{3}$ Pneumology Dept, Hospital Universitario y Politécnico La Fe, Valencia, Spain. ${ }^{4}$ Pneumology Dept, Hospital General de Alicante, Alicante, Spain. ${ }^{5}$ Pneumology Dept, Hospital Clinic-IDIBAPS, Barcelona, Spain. 'Dermatology Dept, Instituto Valenciano de Oncologia, Valencia, Spain. ${ }^{7}$ Dermatology Dept, Hospital de Manises, Valencia, Spain. ${ }^{8}$ Pneumology Dept, Hospital de Valme, Seville, Spain. ${ }^{9}$ Pneumology Dept, Hospital San Pedro de Alcantara, Cáceres, Spain. ${ }^{10}$ Pneumology Dept, Hospital Cruces, Bilbao, Spain. ${ }^{11}$ Pneumology Dept, Hospital Germans Trial i Pujol, Barcelona, Spain. ${ }^{12}$ Pneumology Dept, Hospital de Guadalajara, Guadalajara, Spain. ${ }^{13}$ Pneumology Dept, Fundación Jiménez Diaz, Madrid, Spain. ${ }^{14}$ Pneumology Dept, Hospital de Getafe, Madrid, Spain. ${ }^{15}$ Pneumology Dept, Hospital de la Santa Creu i Sant Pau, Barcelona, Spain. ${ }^{16}$ Pneumology Dept, Hospital 12 de Octubre, Madrid, Spain. ${ }^{17}$ Pneumology Dept, Hospital Consorcio Terrassa, Barcelona, Spain. ${ }^{18}$ Unitat de Biofísica i Bioenginyeria, Facultat de Medicina i Ciències de la Salut, Universitat de Barcelona, Barcelona, Spain. ${ }^{19}$ Dept of Pediatrics, Pritzker School of Medicine, Biological Sciences Division, The University of Chicago, Chicago, IL, USA.

Correspondence: Manuel Sánchez-de-la-Torre, Hospital Arnau de Vilanova-Santa María, IRBLleida, CIBERES, Avda Rovira Roure 80, 25198, Lleida, Spain. E-mail: sanchezdelatorreagmail.com

\section{Introduction}

Obstructive sleep apnoea (OSA) is a prevalent disease which affects $\geqslant 10 \%$ of the adult population [1-3]. OSA is characterised by a repetitive obstruction of the upper airway that leads to intermittent hypoxia and sleep disruption. OSA is associated with an increased risk of cardiovascular and neurocognitive consequences [4].

Cutaneous melanoma is a tumour with a melanocytic origin. The incidence of melanoma has increased worldwide, and 10-30 new melanoma cases per 100000 inhabitants are estimated to occur in Europe and the USA. Melanomas account for $90 \%$ of deaths associated with cutaneous tumours [5]. No effective treatments are available once this malignant tumour has spread [6-8]. The oncogenic transformation of melanocytes to melanoma is the result of complex changes in multiple physiopathological pathways that control cell cycle progression and apoptosis [9]. The identification of treatable diseases that accelerate the progression of melanoma and the pathways involved in this progression can be crucial for reducing the mortality from melanoma.

Recently, OSA has been shown to be associated with a higher cancer prevalence, incidence and mortality [10-12]. Specifically, two recent studies have demonstrated the existence of a relationship between OSA and cutaneous melanoma aggressiveness $[6,13]$. Systemic inflammation due to hypoxia-reoxygenation cycles in OSA may activate diverse physiopathological pathways that enhance tumour progression [14, 15]. Both diseases are associated with elevated circulating levels of adhesion molecules, such as intercellular adhesion molecule (ICAM)-1 [16-18] and vascular cell adhesion molecule (VCAM)-1 [17, 19, 20]; inflammation cytokines, such as vascular endothelial growth factor (VEGF) [21, 22] and interleukin (IL)-8 $[7,16,23]$; and biomarkers of the prognosis and severity of melanoma, such as $\mathrm{S} 100$ calcium binding protein B (S100B) [7, 24] and melanoma inhibitory activity (MIA) [7].

Patients with OSA could be at an increased risk of suffering from neoplastic processes, but to date the specific roles played by OSA have not been clearly established [25]. The objective of the present study was to evaluate the relationship between the biomarkers of carcinogenesis and tumour growth and OSA. Therefore, we measured six biomarkers that represent different physiopathological pathways that may link OSA and melanoma.

\section{Methods \\ Study design}

This ancillary, observational, prospective, cross-sectional and multicentre clinical study was conducted in 29 teaching hospitals in Spain and included patients with cutaneous melanoma. Briefly, the relationship between the presence and severity of sleep disordered breathing and melanoma aggressiveness was analysed. This study was approved by the ethics committees of each of the participating hospitals. All participants provided signed informed consent to participate in the study.

\section{Patient selection}

The patients were consecutively recruited at the dermatology or oncology units of each participating centre. The patients were initially eligible for participation in the study if they had a diagnosis of invasive cutaneous melanoma, were aged $>18$ years and signed the informed consent to participate in the study. The initial exclusion criteria included primary unknown melanoma, extracutaneous melanoma, in situ melanoma, pregnancy, daytime respiratory or heart failure and current or previous use of continuous positive airway pressure therapy (CPAP). Because the metastatic status of patients may strongly 
influence the levels of the circulating biomarkers of cancer, those patients with metastatic status were excluded (figure 1).

\section{Sleep studies}

The patients included in the study underwent polygraphy for a maximum of 6 months after the cutaneous melanoma diagnosis at the sleep unit of each centre. The respiratory polygraphy included a continuous recording of the oronasal flow and pressure, heart rate, thoracic and abdominal respiratory movements and arterial oxygen saturation $\left(\mathrm{SaO}_{2}\right)$. Trained personnel scored the polygraphy data manually. Apnoea was defined as an interruption of the oronasal airflow for $>10$ s. Hypopnoea was defined as a 30-90\% reduction in the oronasal airflow for $>10 \mathrm{~s}$, which was associated with an oxygen desaturation $\geqslant 3 \%$. The apnoea-hypopnoea index (AHI) was defined as the number of apnoea events plus hypopnoea events per hour during the recording; in addition, the percentage of the recording time with an $\mathrm{SaO}_{2}<90 \%$ was recorder. In addition, the baseline saturation, mean saturation, minimum saturation and oxygen desaturation index (ODI) at $3 \%$ and $4 \%$ were measured.

The patients were divided into the following three groups: the non-OSA group (AHI $0-5$ events.h ${ }^{-1}$ ), the mild sleep apnoea group (AHI 5-15 events $\mathrm{h}^{-1}$ ) and the moderate-severe sleep apnoea group (AHI $>15$ events $\left.\cdot h^{-1}\right)$.

\section{Evaluation of the growth rate of cutaneous melanoma}

Cutaneous melanoma growth rate was calculated as the Breslow index (in millimetres) divided by the difference between the date of the excision of the tumour and the date on which patients noticed changes suggestive of malignant transformation in a stable, pre-existing lesion or the time they noticed the appearance of a new and changing lesion [6]. Cutaneous melanoma growth rate was categorised as follows: slow-intermediate $\left(<0.49 \mathrm{~mm} \cdot \mathrm{month}^{-1}\right)$ and fast $\left(\geqslant 0.5 \mathrm{~mm} \cdot \mathrm{month}^{-1}\right)$ [26].

\section{Measurements of circulating molecules}

Fasting venous blood samples were drawn between 08:00 h and 09:00 h. The blood samples were centrifuged to separate the serum, and all specimens were immediately aliquoted, frozen and stored at $-80^{\circ} \mathrm{C}$.

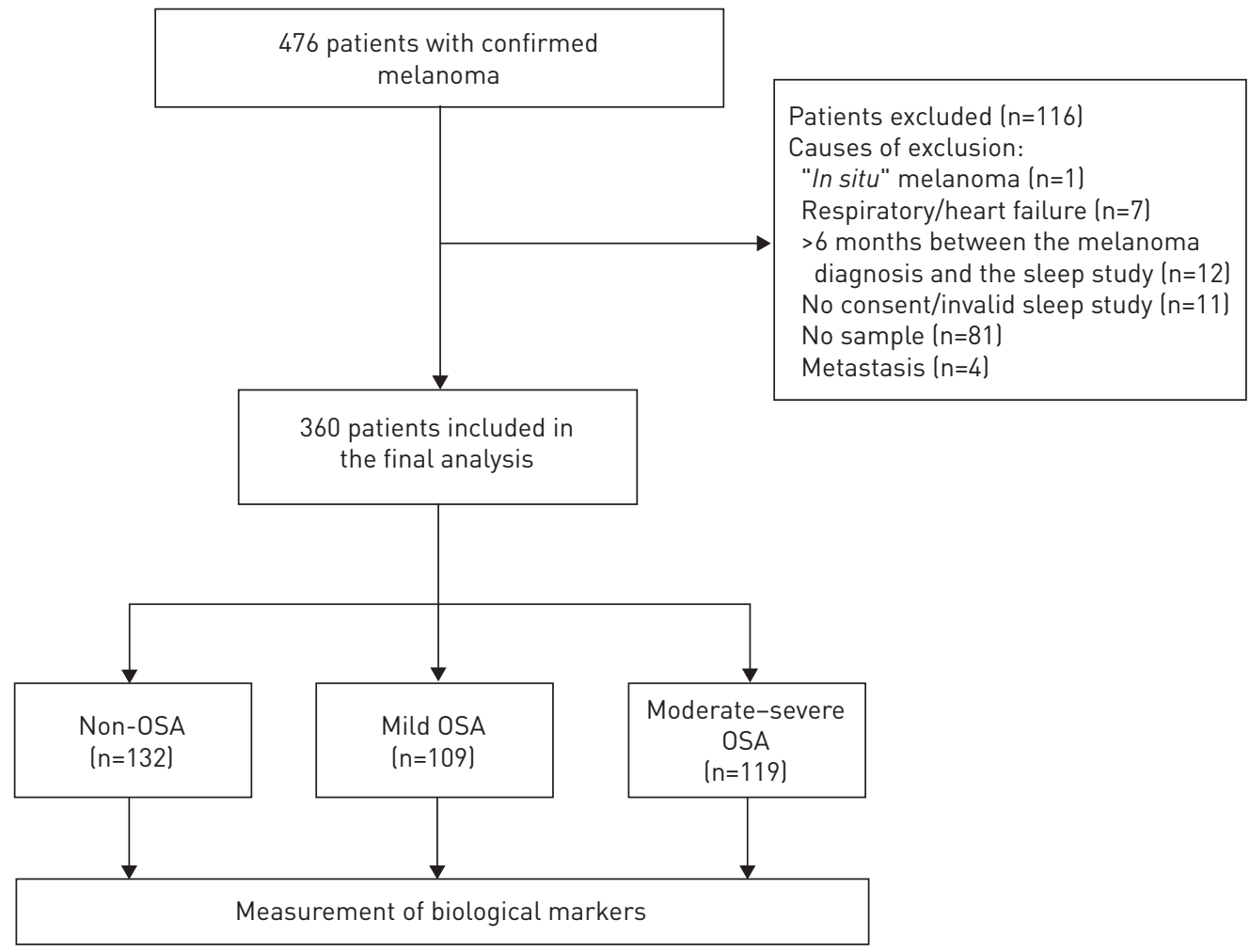

FIGURE 1 Flowchart of the study. OSA: obstructive sleep apnoea. 
Two different methods, i.e. multiplex analyses and traditional ELISAs were used to quantify the circulating molecules. Briefly, Q-Plex ELISA (Quansys Biosciences, Logan, UT, USA) technology allows for the simultaneous measurement and detection of multiple proteins at the microscale level. This technology involves an array of quantitative sandwich ELISAs that use standard curves for data interpolation. Using the sandwich ELISAs, capture antibodies are spotted into a 96-well microtitre plate and bind to target proteins in the sample, and secondary or detection antibodies bind to the different epitopes on the target proteins. Streptavidin-horseradish peroxidase is preconjugated to secondary antibodies and used to generate a signal. The measured signal is proportional to the amount of the target protein in the sample.

The two multiplexed proteins (including VEGF and IL-8) were analysed using the Q-View Imager. Individual ELISAs were performed to detect VCAM-1, ICAM-1, MIA and S100B following the manufacturer's recommendations. For each assay, standard curves were generated to determine the concentrations of the proteins. The sensitivity and ELISA trademark for each protein are presented in online supplementary table E1.

\section{Measurements of molecules in post-excisional melanoma tumour tissues}

The expression levels of VEGF and the levels of hypoxia-inducible factor (HIF)-1 $\alpha$ were measured and previously reported in this cohort of patients [27] to determine whether assessments of the abundance of these biomarkers in post-excisional melanoma tumour tissues may lead to a more accurate determination of tumour aggressiveness. For the present study, we evaluated the association of the expression levels of these biomarkers with OSA severity. The levels of VEGF and HIF-1 $\alpha$ were assessed using immunohistochemistry on $4-\mu \mathrm{m}$-thick sections of formalin-fixed, paraffin-embedded melanoma samples. Immunostaining was performed on a Leica Bond-III stainer (Leica Biosystems, Newcastle, UK) using the Leica Bond Polymer Refine Kit (Leica Biosystems). Melanoma samples were stained with a 1:2 dilution of anti-VEGF prediluted antibody (ab27620; Abcam, Cambridge, UK) and with a 1:100 dilution of anti-HIF-1 $\alpha$ antibody (ab51608; Abcam). The staining results were analysed independently by two expert pathologists who were blinded to the staging and clinical features of the patients. VEGF-specific staining was scored considering both the percentage of the positive $(<25 \%, 25-50 \%, 51-75 \%$ and $>75 \%)$ and the expression intensity (negative-low or high). HIF-1 $\alpha$-specific nuclear staining was scored considering the presence or absence of positive cells.

\section{Statistical analysis}

The quantitative variables are expressed as median (interquartile range (IQR)), since these variables were non-normally distributed. Absolute and relative frequencies were used to describe the qualitative variables. We performed a bivariate analysis to analyse the studied variables according to the AHI group. The Kruskal-Wallis test was performed to compare the quantitative variables, and Pearson's Chi-squared or Fisher's exact test was performed to compare the qualitative variables. A logistic regression model (adjusted for age, sex and body mass index (BMI)) was fitted for each of the ELISAs as the explanatory variable(s), and the AHI, which was categorised by severity (non-OSA, mild OSA and moderate-severe OSA), was the dependent variable. The calibration was assessed using the Hosmer-Lemeshow goodness-of-fit test. All tests were two-tailed, and a statistical significance level of 0.05 was used. The statistical analyses were performed using $\mathrm{R}$ version 3.3.1.

\section{Results}

\section{Patient characteristics}

Of the 476 patients who were initially recruited, 360 patients with a diagnosis of cutaneous melanoma who underwent a sleep study and provided at least one serum sample were included in the final analysis (figure 1). The clinical characteristics and demographic variables of the patients are shown in table 1. The study population included middle-aged males and females who were overweight and had mild sleep apnoea (AHI 8.55 events $\cdot h^{-1}$, range 2.80-19.5 events $\cdot h^{-1}$ ).

\section{Analysis of serum markers}

The results of the measurements of the circulating levels of VEGF, IL-8, ICAM-1, VCAM-1, S100B and MIA are presented in figure 2. The serum levels of IL-8, ICAM-1, S100B and MIA were similar among the non-OSA, mild OSA and moderate-severe OSA patients $(\mathrm{p}=0.436, \mathrm{p}=0.259, \mathrm{p}=0.560$ and $\mathrm{p}=0.328$, respectively). These biomarkers, which are related to inflammation, progression and prognosis in cutaneous melanoma, were not significantly related to the severity of OSA. In contrast, the serum levels of VEGF exhibited an increased tendency $(\mathrm{p}=0.165)$ in both the mild OSA (odds ratio (OR) 1.26, 95\% CI 0.70-2.28) and moderate-severe sleep apnoea (OR 1.61, 95\% CI 0.85-3.06) groups (figure 2 and table 2). Similarly, we observed that the serum levels of VCAM-1 were significantly higher in the sleep apnoea patients than those in the non-OSA patients, for mild OSA (OR 2.07, 95\% CI 1.12-3.89; $\mathrm{p}=0.021$ ) and for 
TABLE 1 Anthropometric and clinical characteristics of the study groups

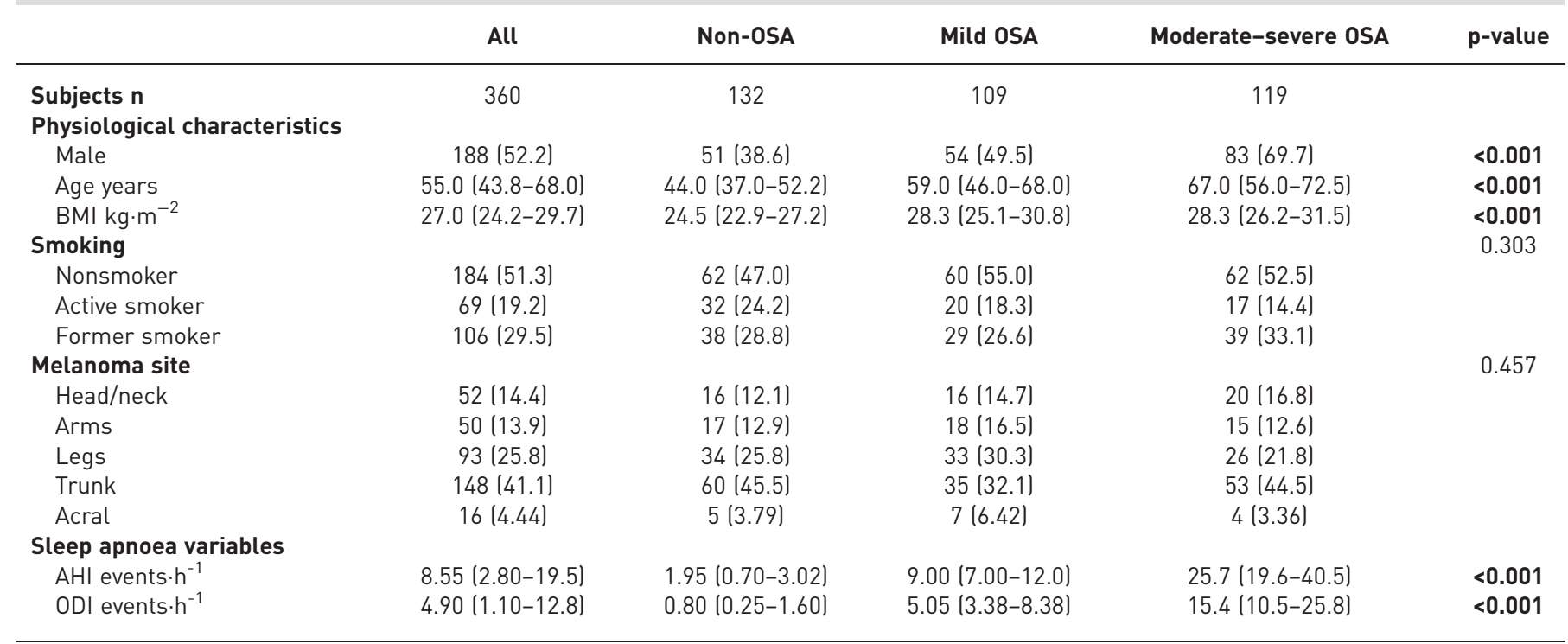

Data are presented as $\mathrm{n}(\%)$ or median (interquartile range), unless otherwise stated. OSA: obstructive sleep apnoea; BMI: body mass index; AHI: apnoea-hypopnoea index; ODI: oxygen desaturation index. Statistically significant $p$-values $(p<0.05)$ are denoted in bold.

moderate-severe OSA (OR 2.35, 95\% CI 1.20-4.66; $\mathrm{p}=0.013$ ) after adjusting for BMI, age and sex (table 2 and figure 2). Similar results were obtained by comparing the groups according to the ODI terciles (data not shown).

\section{Analysis of tumour tissues samples}

Assessment of the levels of VEGF and HIF-1 $\alpha$ expression in the tissue samples from the resected tumour are presented in table 3. Although a tendency was observed in the HIF-1 $\alpha$ proportion of positive-stained tumours in patients with moderate-severe OSA, the adjusted analysis reported similar proportions among non-OSA patients, mild OSA (OR 1.52, 95\% CI 0.64-3.75; p=0.35) and moderate-severe OSA (OR 1.82, 95\% CI 0.73-4.77; $\mathrm{p}=0.21$ ) (table 3). For VEGF, the proportion of stained cells in tumour tissues samples was $>75 \%$ for the three groups. Odds ratios were obtained for VEGF expression in tissue samples of the resected tumour and no significant differences emerged between groups (table 3 ).

\section{Relationship between cutaneous melanoma growth rate and VCAM-1}

VCAM-1 levels were categorised as follows according to the mean levels: high levels of VCAM-1 (above the mean) and low levels of VCAM-1 (below the mean). It was observed a tendency of high serum levels of VCAM-1 positively associated with a fast cutaneous melanoma growth rate (figure 3) (1.59, 95\% CI $0.91-2.76)$, but this association did not reach statistical significance $(\mathrm{p}=0.112)$.

\section{Discussion}

In the present study we described how in a cohort of patients with cutaneous melanoma, the OSA severity was related to the serum levels of the VCAM-1 tumour adhesion-related biomarker, but not to the serum levels of markers related with tumour aggressiveness, such as VEGF, IL-8, ICAM-1, S100B and MIA, or levels of VEGF and HIF-1 $\alpha$ expression in tissue samples of the tumour.

The increase in the circulating levels of ICAM-1 and the correlations between ICAM-1 and AHI have been previously reported $[16,19,28]$. The circulating levels of VEGF are frequently increased in OSA patients and play a role in the regulation of tissue oxygen delivery $[22,28]$. The levels of IL-8 have been reported to be higher in OSA patients than those in healthy controls $[16,29]$, while the S100B levels are increased in OSA patients but do not correlate with the severity of the disease $[24,30]$. We only observed increased levels of VCAM-1 and moderate increased levels of VEGF, which may be due to the differences between the populations since our cohort included patients with cutaneous melanoma and OSA; the cutaneous melanoma effect, that is independently related with the increase of VEGF [31, 32], together with IL- 8 [28, 32], ICAM-1 [18, 33], S100B [7, 34, 35] and MIA [7, 35, 36], could mask the OSA effect on these proteins, which may explain why we did not observe the previously reported differences in these biomarkers in the patients with OSA. Although the possibility exists that the presence of cutaneous 

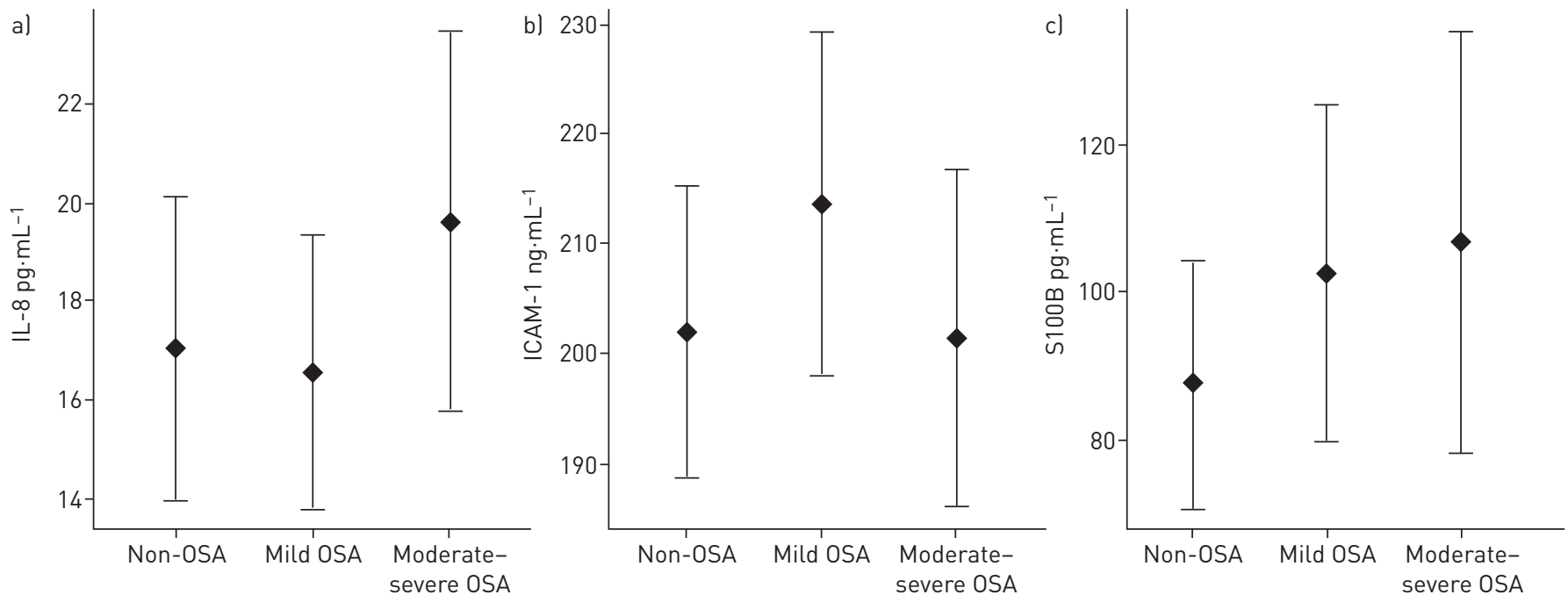

Apnoea-hypopnoea index

Apnoea-hypopnoea index

Apnoea-hypopnoea index

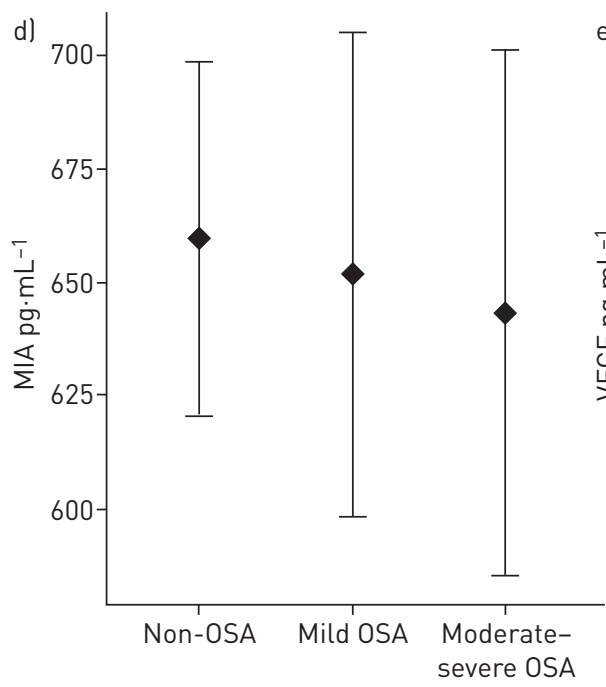

Apnoea-hypopnoea index e)

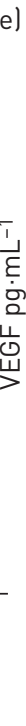

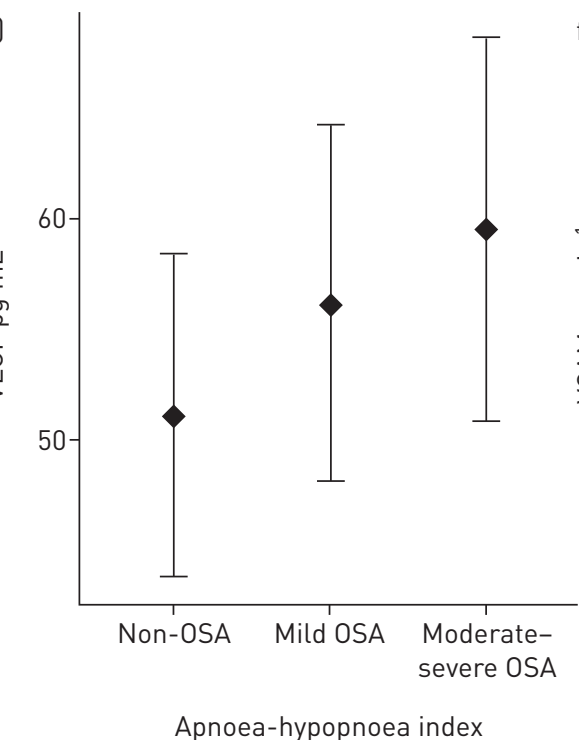

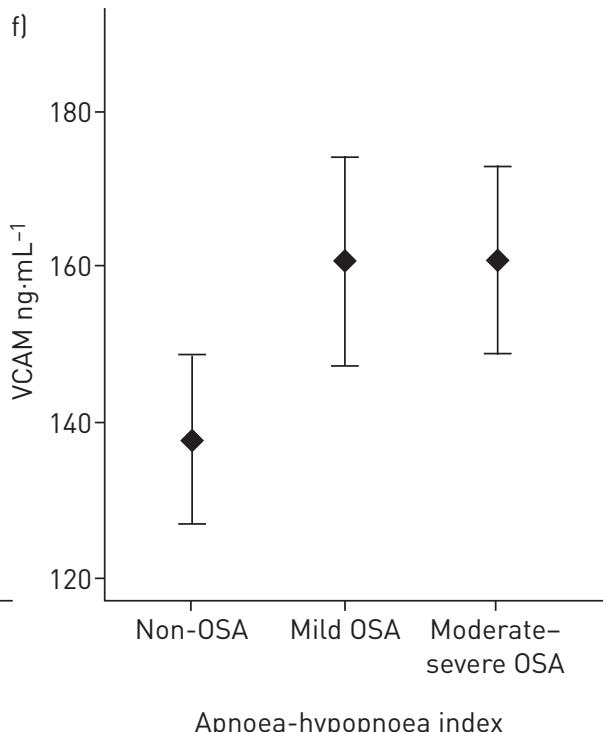

FIGURE 2 Circulating levels of biomarkers. a) Interleukin (IL)-8; b) intracellular adhesion molecule (ICAM)-1; c) S100 calcium-binding protein B (S100B); d) melanoma inhibitory activity (MIA); e) vascular endothelial growth factor (VEGF); f) vascular cell adhesion molecule (VCAM). Data are presented as median (interquartile range). OSA: obstructive sleep apnoea.

melanoma may exert a potential ceiling effect on the contributions of OSA to the biomarkers of interest, the study was not designed to address this issue, which therefore must remain speculative at present. In the present study, we were not able to evaluate the specific separate effects of OSA and cutaneous melanoma. This further buttresses the need to disassociate the relative contributions of OSA and cutaneous melanoma to biomarkers such as those explored in the study. However, the study was not designed to address this specific possibility. Indeed, we would have needed to include two additional matched groups (no cutaneous melanoma and no OSA; and OSA alone in the absence of cutaneous melanoma), and then further evaluate the effect of treating OSA on the biomarkers of interest to segregate the independent and overlapping contributions of cutaneous melanoma and OSA to these biomarkers.

Several pathophysiological mechanisms, including key intermediate molecules, link OSA to the aggressiveness of cancer. Patients with OSA have higher serum levels of VCAM-1 than healthy controls $[17,19,28]$, which is primarily due to hypoxic stress. OSA could enhance the aggressiveness of cutaneous melanoma and increase the levels of VCAM-1.

The secreted form of VCAM-1 is due to proteolytic cleavage upon its release from the cell surface via the activity of neutrophil-derived serine proteases. VCAM-1 is mainly involved in leukocyte transendothelial 


\begin{tabular}{|c|c|c|}
\hline & Odds ratio $(95 \% \mathrm{Cl})^{\#}$ & $\mathrm{p}$-value \\
\hline \multicolumn{3}{|l|}{ VEGF } \\
\hline Mild OSA & $1.26(0.70-2.00)$ & 0.431 \\
\hline Moderate-severe OSA & $1.61(0.85-3.06)$ & 0.143 \\
\hline \multicolumn{3}{|l|}{ IL-8 } \\
\hline Mild OSA & $0.75(0.42-1.35)$ & 0.345 \\
\hline Moderate-severe OSA & $1.04(0.55-1.97)$ & 0.902 \\
\hline \multicolumn{3}{|l|}{ ICAM } \\
\hline Mild OSA & $1.11(0.62-1.96)$ & 0.724 \\
\hline Moderate-severe OSA & $0.69(0.37-1.30)$ & 0.253 \\
\hline \multicolumn{3}{|l|}{ VCAM } \\
\hline Mild OSA & $2.07(1.12-3.89)$ & 0.021 \\
\hline Moderate-severe OSA & $2.35(1.20-4.66)$ & 0.013 \\
\hline \multicolumn{3}{|l|}{ S100B } \\
\hline Mild OSA & $1.27(0.70-2.34)$ & 0.434 \\
\hline Moderate-severe OSA & $0.94(0.49-1.76)$ & 0.883 \\
\hline \multicolumn{3}{|l|}{ MIA } \\
\hline Mild OSA & $1.20(0.63-2.31)$ & 0.582 \\
\hline Moderate-severe OSA & $0.70(0.34-1.40)$ & 0.312 \\
\hline \multicolumn{3}{|c|}{$\begin{array}{l}\text { VEGF: vascular endothelial growth factor; OSA: obstructive sleep apnoea; IL: interleukin; ICAM: } \\
\text { intracellular adhesion molecule; VCAM: vascular cell adhesion molecule } 1 ; \mathrm{S} 100 \mathrm{~B} \text { : S100 calcium-binding } \\
\text { protein B; MIA: melanoma inhibitory activity. ": odds ratios were calculated using a logistic regression; the } \\
\text { response variables were the individual biomarkers, and the explanatory variables were the categories of } \\
\text { OSA. The model was adjusted for sex, age and body mass index. Statistically significant p-values (p<0.05) } \\
\text { are denoted in bold. }\end{array}$} \\
\hline
\end{tabular}

migration and leukocyte retention into tissues [37]. VCAM-1 plays a central role in the recruitment of inflammatory cells, and its expression is rapidly induced by proinflammatory cytokines, such as VEGF [38]. VCAM-1 plays important roles due to its multiple functionalities in directing the spread of tumours, the formation of metastatic niches and supporting the angiogenic process [20]. Thus, OSA due to hypoxic stress could elevate the serum levels of VEGF and stimulate the expression of VCAM-1, which promotes tumorigenesis. This hypothesis is supported by the relationship between the VCAM-1 circulating levels and the growth rate of melanoma. Even though the result was not statistically significant, a tendency to a positive association could exist, and should be further explored.

For the first time, a biomarker for tumorigenesis has been reported to be elevated in patients with OSA who already suffer from cutaneous melanoma. These findings could highlight the importance of VCAM-1 in tumorigenesis in patients with OSA, as a possible pathway that relates OSA with cutaneous melanoma. In the present study, we observed a tendency toward increased HIF- $1 \alpha$ in post-excisional melanoma tumour tissues as the severity of OSA increased, although the adjusted analysis yielded similar expression

TABLE 3 Adjusted logistic regression models of the expression of the biomarkers in the resected tumour samples in the groups analysed

\begin{tabular}{|c|c|c|}
\hline & Odds ratio $(95 \% \mathrm{CI})^{\#}$ & $p$-value \\
\hline \multicolumn{3}{|l|}{ VEGF } \\
\hline Mild OSA & $1.69(0.85-3.39)$ & 0.14 \\
\hline Moderate-severe OSA & $1.35(0.64-2.85)$ & 0.43 \\
\hline \multicolumn{3}{|l|}{ 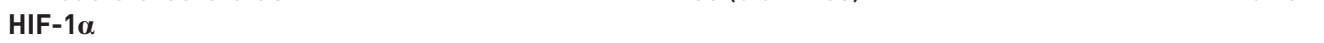 } \\
\hline Mild OSA & $1.52(0.64-3.75)$ & 0.35 \\
\hline Moderate-severe OSA & $1.82(0.73-4.77)$ & 0.21 \\
\hline \multicolumn{3}{|c|}{$\begin{array}{l}\text { VEGF: vascular endothelial growth factor; OSA: obstructive sleep apnoea; HIF: hypoxia inducible factor. } \\
\# \text { : odds ratios were calculated using a logistic regression; the response variables were the individual } \\
\text { biomarkers, and the explanatory variables were the categories of OSA. The model was adjusted for sex, } \\
\text { age and body mass index. }\end{array}$} \\
\hline
\end{tabular}


FIGURE 3 Relationship between the circulating levels of vascular cell adhesion molecule (VCAM)-1 and the cutaneous melanoma growth rate. Data are presented as median (interquartile range).

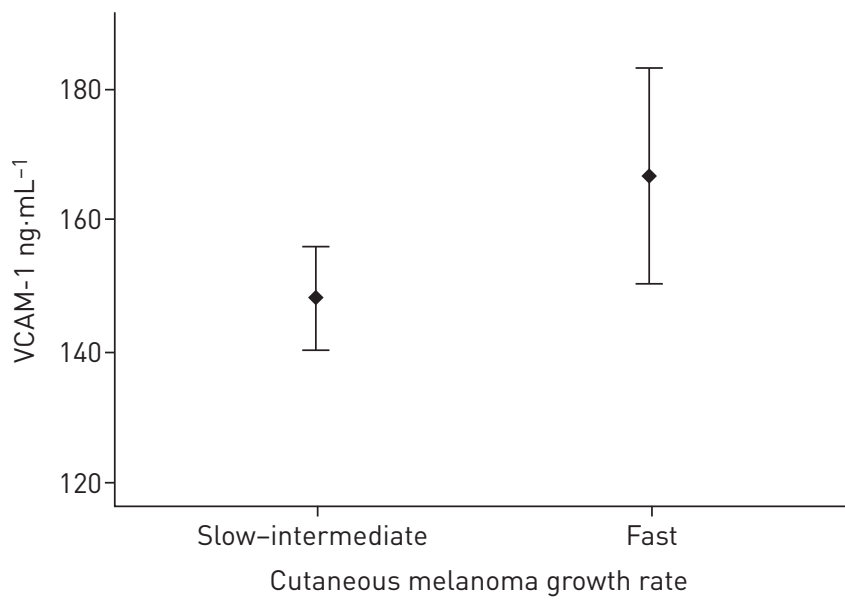

levels between mild OSA and moderate/severe OSA. No significant association was observed between VEGF levels in tumour samples and OSA severity. The fact that the biomarkers that were significantly associated with OSA in patients with cutaneous melanoma consisted of levels measured in circulating blood samples may be relevant as far as their clinical utility, considering their accessibility. The circulating levels of VEGF and VCAM-1 could be helpful in the identification of patients with melanoma and OSA that are at a higher risk of carcinogenesis and tumour growth. Additionally, measuring the VEGF and VCAM-1 circulating levels is important not only in patients with cutaneous melanoma but also in patients with only OSA because these biomarkers play an important role in the initial stages of tumorigenesis, and OSA patients with elevated serum levels of VCAM-1 and VEGF could be more likely to suffer from tumorigenesis. These findings could support the hypothesis that treating patients with cutaneous melanoma and OSA, with increased levels of circulating VEGF and VCAM-1 could reduce tumorigenesis and improve tumour outcomes.

Intermittent hypoxia and arousal, the hallmark features of OSA, are recognised as the major pathophysiological consequences of OSA that promote the activation of intermediate mechanisms that associate OSA with its deleterious consequences and may contribute to an increased cancer risk [25]. Consistent with the evidence from laboratory and animal experiments [39], previous observational trials have reported cancer incidence to be augmented with increasing OSA levels [40,41]. These observational studies suggested an association between nocturnal hypoxaemia and cancer mortality in patients with OSA. Notably, in patients who remained untreated, the association between the hypoxaemic index and cancer incidence was slightly stronger. The application of treatment that allows the abolition of the apnoea may contribute to the reduction in these angiogenic biomarkers associated with cancer. Although there are data that clearly suggest an association between sleep apnoea and cancer risk, there is an absolute lack of evidence of the possible positive effect of CPAP treatment on decreasing tumour aggressiveness in patients with OSA. Although the effects of CPAP treatment in patients with OSA and cancer have not been explored, basic research studies have identified the role of CPAP treatment in mechanisms related to tumorigenesis. Indeed, GHARIB et al. [42] studied the whole-genome expression of peripheral blood leucocytes in 18 patients with OSA before and after 1 month of CPAP treatment. Gene-set enrichment analysis comparing leucocytes in each patient from pre- and post-CPAP treatment indicated that some gene sets involved in neoplastic processes were downregulated by OSA treatment. Moreover, HERNÁNDEZ-JIMÉNEZ et al. [43] demonstrated that OSA induces changes in the functionality of circulating monocytes and natural killer cells, which could favour tumour immune escape. This finding was not observed in CPAP-treated patients. Finally, AlmEndros et al. [44] evaluated in a murine model whether the chronic intermittent hypoxia that characterises OSA leads to release of tumour-promoting exosomes in the circulation. These investigators observed that the exosomes released during intermittent hypoxia mimicking OSA increase the malignancy properties of TC1 lung tumour cells in vitro. In addition, in this study, the authors evaluated exosomes from 10 adult human patients with OSA for 6 weeks before and after CPAP treatment and reported that exosomes from patients with OSA increase proliferation and migration when compared with the same patients after 6 weeks of adherent CPAP treatment.

CPAP treatment is the gold standard for treating OSA and should be considered the first-line therapeutic option for OSA. Mandibular advancement devices have also been considered for OSA treatment, particularly in mild-moderate OSA [45]. Effective OSA treatment in patients with cutaneous melanoma and OSA may contribute to the reduction of the levels of markers related to cancer. Because of the lack of 
evidence of the possible positive effects of CPAP treatment in cancer progression this possibility should be considered speculative at present. Further studies are necessary to evaluate the effect of CPAP treatment in tumorigenic biomarkers and the prognosis of patients with OSA and cancer.

The main strengths of the study are related to its prospective nature and the relatively large cohort size that enables appropriate gauging of the potential effects of OSA severity on biomarkers related with carcinogenesis and tumour growth. Nevertheless, the present study has several limitations that deserve comment. First, sleep habits and shift work, variables that are related to cancer, were not reported [12]. Second, the results of this study are specifically related to cutaneous melanoma. Because of that, the relationship observed for VCAM-1 and VEGF could not be present in other types of cancer. Third, the present study evaluates the relationship between the severity of OSA and the levels of cancer-related biomarkers in patients with cutaneous melanoma; however, no information is available regarding the effect of CPAP treatment on these biomarkers.

In conclusion, our study suggests that in patients with cutaneous melanoma, OSA is independently associated with high levels of circulating VCAM-1 tumour adhesion-related biomarker, but not to circulating levels of markers related with tumour aggressiveness. The activation of pathways related with tumour adhesion could be related with the observed relationship between OSA severity and melanoma. Further studies should be explored to identify the intermediate pathways linking OSA severity and cancer.

\section{References}

1 Durán J, Esnaola S, Rubio R, et al. Obstructive sleep apnea-hypopnea and related clinical features in a population-based sample of subjects aged 30 to 70 yr. Am J Respir Crit Care Med 2001; 163: 685-689.

2 Heinzer R, Vat S, Marques-Vidal P, et al. Prevalence of sleep-disordered breathing in the general population: the HypnoLaus study. Lancet Respir Med 2015; 3: 310-318.

3 Peppard PE, Young T, Barnet JH, et al. Increased prevalence of sleep-disordered breathing in adults. Am J Epidemiol 2013; 177: 1006-1014.

4 Sánchez-de-la-Torre M, Campos-Rodriguez F, Barbé F. Obstructive sleep apnoea and cardiovascular disease. Lancet Respir Med 2013; 1: 61-72.

5 Garbe C, Peris K, Hauschild A, et al. Diagnosis and treatment of melanoma. European consensus-based interdisciplinary guideline - update 2016. Eur J Cancer 2016; 63: 201-217.

6 Martínez-Garćia M-Á, Martorell-Calatayud A, Nagore E, et al. Association between sleep disordered breathing and aggressiveness markers of malignant cutaneous melanoma. Eur Respir J 2014; 43: 1661-1668.

7 Alegre E, Sammamed M, Fernández-Landázuri S, et al. Circulating biomarkers in malignant melanoma. Adv Clin Chem 2015; 69: 47-89.

$8 \quad$ Garbe C, Leiter U. Melanoma epidemiology and trends. Clin Dermatol 2009; 27: 3-9.

9 Hulstaert E, Brochez L, Volders P, et al. Long non-coding RNAs in cutaneous melanoma: clinical perspectives. Oncotarget 2017; 8: 43470-43480.

10 Gozal D, Ham SA, Mokhlesi B. Sleep apnea and cancer: analysis of a nationwide population sample. Sleep 2016; 39: $1493-1500$.

11 Martínez-García MÁ, Campos-Rodríguez F, Almendros I, et al. Relación entre apnea del sueño y cancer. [Relationship between sleep apnea and cancer]. Arch Bronconeumol 2015; 51: 456-461.

12 Santamaria-Martos F, Sánchez-de-la-Torre M, Martínez-García MA. Sleep and cancer: clinical studies and opportunities for personalized medicine. Curr Sleep Med Reports 2017; 3: 11-21.

13 Almendros I, Montserrat JM, Torres M, et al. Intermittent hypoxia increases melanoma metastasis to the lung in a mouse model of sleep apnea. Respir Physiol Neurobiol 2013; 186: 303-307.

14 Gozal D, Farré R, Nieto FJ. Putative links between sleep apnea and cancer from hypotheses to evolving evidence. Chest 2015; 148: 1140-1147.

15 Martínez-García MÁ, Campos-Rodriguez F, Barbé F. Cancer and OSA: current evidence from human studies. Chest 2016; 150: 451-463.

16 Carpagnano GE, Spanevello A, Sabato R, et al. Systemic and airway inflammation in sleep apnea and obesity: the role of ICAM-1 and IL-8. Transl Res 2010; 155: 35-43.

17 Ohga E, Nagase T, Tomita T, et al. Increased levels of circulating ICAM-1, VCAM-1, and L-selectin in obstructive sleep apnea syndrome. J Appl Physiol 1999; 87: 10-14.

18 Kageshita T, Yoshii A, Kimura T, et al. Clinical relevance of ICAM-1 expression in primary lesions and serum of patients with malignant melanoma. Cancer Res 1993; 53: 4927-4932.

19 Ursavaş A, Karadağ M, Rodoplu E, et al. Circulating ICAM-1 and VCAM-1 levels in patients with obstructive sleep apnea syndrome. Respiration 2007; 74: 525-532.

20 Schlesinger M, Bendas G. Vascular cell adhesion molecule-1 (VCAM-1) - an increasing insight into its role in tumorigenicity and metastasis. Int J Cancer 2015; 136: 2504-2514.

21 Ascierto PA, Leonardi E, Ottaiano A, et al. Prognostic value of serum VEGF in melanoma patients: a pilot study. Anticancer Res 24: 4255-4258.

22 Gozal D, Lipton AJ, Jones KL. Circulating vascular endothelial growth factor levels in patients with obstructive sleep apnea. Sleep 2002; 25: 59-65.

23 Ugurel S, Rappl G, Tilgen W, et al. Increased serum concentration of angiogenic factors in malignant melanoma patients correlates with tumor progression and survival. J Clin Oncol 2001; 19: 577-583.

24 Duru S, Hikmet Firat I, Colak N, et al. Serum S100B protein: a useful marker in obstructive sleep apnea syndrome. Neurol Neurochir Pol 2012; 46: 450-455.

25 Gozal D, Farré R, Nieto FJ. Obstructive sleep apnea and cancer: epidemiologic links and theoretical biological constructs. Sleep Med Rev 2016; 27: 43-55. 
26 Martorell-Calatayud A, Nagore E, Botella-Estrada R, et al. Defining fast-growing melanomas: reappraisal of epidemiological, clinical, and histological features. Melanoma Res 2011; 21: 131-138.

27 Martínez-García MÁ, Riveiro-Falkenbach E, Rodríguez-Peralto JL, et al. A prospective multicenter cohort study of cutaneous melanoma: clinical staging and potential associations with HIF-1 $\alpha$ and VEGF expressions. Melanoma Res 2017; 27: 558-564.

28 Nadeem R, Molnar J, Madbouly EM, et al. Serum inflammatory markers in obstructive sleep apnea: a meta-analysis. J Clin Sleep Med 2013; 9: 1003-1012.

29 Ohga E, Tomita T, Wada H, et al. Effects of obstructive sleep apnea on circulating ICAM-1, IL-8, and MCP-1. J Appl Physiol 2003; 94: 179-184.

30 Traxdorf M, Wendler O, Tziridis K, et al. S100B in serum and saliva: a valid invasive or non-invasive biomarker in obstructive sleep apnea? Eur Rev Med Pharmacol Sci 2016; 20: 4766-4774.

31 Tas F, Duranyildiz D, Oguz H, et al. Circulating levels of vascular endothelial growth factor (VEGF), matrix metalloproteinase-3 (MMP-3), and BCL-2 in malignant melanoma. Med Oncol 2008; 25: 431-436.

32 Yurkovetsky ZR, Kirkwood JM, Edington HD, et al. Multiplex analysis of serum cytokines in melanoma patients treated with interferon- $\alpha 2$ b. Clin Cancer Res 2007; 13: 2422-2428.

33 Yasasever V, Tas F, Duranyildiz D, et al. Serum levels of the soluble adhesion molecules in patients with malignant melanoma. Pathol Oncol Res 2000; 6: 42-45.

34 Karonidis A, Mantzourani M, Gogas H, et al. Serum S100B levels correlate with stage, N status, mitotic rate and disease outcome in melanoma patients independent to LDH. J BUON 2017; 22: 1296-1302.

35 Garbe C, Leiter U, Ellwanger U, et al. Diagnostic value and prognostic significance of protein S-100 3 , melanoma-inhibitory activity, and tyrosinase/MART-1 reverse transcription-polymerase chain reaction in the follow-up of high-risk melanoma patients. Cancer 2003; 97: 1737-1745.

36 Odashiro M, Hans Filho G, Pereira PR, et al. Melanoma inhibitory activity in Brazilian patients with cutaneous melanoma. An Bras Dermatol 2015; 90: 327-332.

37 Wang P-C, Weng C-C, Hou Y-S, et al. Activation of VCAM-1 and its associated molecule CD44 leads to increased malignant potential of breast cancer cells. Int J Mol Sci 2014; 15: 3560-3579.

38 Ding Y-B, Chen G-Y, Xia J-G, et al. Association of VCAM-1 overexpression with oncogenesis, tumor angiogenesis and metastasis of gastric carcinoma. World J Gastroenterol 2003; 9: 1409-1414.

39 Almendros I, Montserrat JM, Ramírez J, et al. Intermittent hypoxia enhances cancer progression in a mouse model of sleep apnoea. Eur Respir J 2012; 39: 215-217.

40 Martínez-García MA, Campos-Rodriguez F, Durán-Cantolla J, et al. Obstructive sleep apnea is associated with cancer mortality in younger patients. Sleep Med 2014; 15: 742-748.

41 Nieto FJ, Peppard PE, Young T, et al. Sleep-disordered breathing and cancer mortality: results from the Wisconsin Sleep Cohort Study. Am J Respir Crit Care Med 2012; 186: 190-194.

42 Gharib SA, Seiger AN, Hayes AL, et al. Treatment of obstructive sleep apnea alters cancer-associated transcriptional signatures in circulating leukocytes. Sleep 2014; 37: 709-714.

43 Hernández-Jiménez E, Cubillos-Zapata C, Toledano V, et al. Monocytes inhibit NK activity via TGF- $\beta$ in patients with obstructive sleep apnoea. Eur Respir J 2017; 49: 1602456.

44 Almendros I, Khalyfa A, Trzepizur W, et al. Tumor cell malignant properties are enhanced by circulating exosomes in sleep apnea. Chest 2016; 150: 1030-1041.

45 Jayesh SR, Bhat W. Mandibular advancement device for obstructive sleep apnea: an overview. J Pharm Bioallied Sci 2015; 7: S223-S225. 